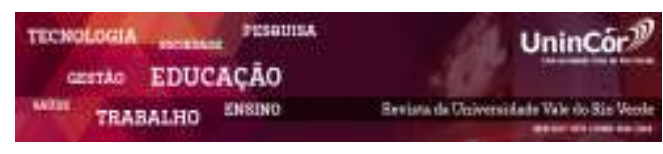

Revista da Universidade Vale do Rio Verde ISSN: 1517-0276 / EISSN: 2236-5362 Vol. $16 \mid$ n. 2| Ano 2018

Rafael Rudolfo Kreutz Universidade Federal de Santa Maria rafael.kreutz@gmail.com

Nelson Guilherme Machado Pinto Universidade Federal de Santa Maria nelguimachado@hotmail.com

\section{TRANSPARÊNCIA NO SETOR PÚBLICO: UMA ANÁLISE DAS PUBLICAÇÕES CIENTÍFICAS INTERNACIONAIS}

\section{RESUMO}

O presente estudo procura identificar um panorama sobre a temática da transparência pública, a qual tem se destacado na mídia nos últimos anos e ainda apresenta um conceito amplamente em discussão na literatura. Desse modo, o objetivo do estudo é verificar a evolução do tema através da publicação de artigos em periódicos internacionais sobre transparência no setor público no período de 2007 a 2017. A pesquisa foi estabelecida através da busca de publicações em periódicos com base no Scopus. O processo de análise foi realizado por meio de um processo de organização, classificação e análise de um conjunto de informações e dados bibliométricos. Para tanto, essa pesquisa é delineada como descritiva, de análise bibliométrica e qualitativaquantitativa. Foram encontrados 540 artigos com predomínio massivo de trabalhos publicados nos Estados Unidos, seguido pela Espanha e Reino Unido, em segundo e terceiros lugares respectivamente. Além disso, os resultados demonstram que a literatura mundial acerca da temática transparência pública se encontra consolidada, diferentemente da literatura nacional que ainda necessita ter maiores avanços da pesquisa nessa área.

Palavras-chave: Transparência. Setor Público. Publicações Científicas. Governança. Bibliometria.

\section{TRANSPARENCY IN THE PUBLIC SECTOR: AN ANALYSIS OF INTERNATIONAL SCIENTIFIC PUBLICATIONS}

\begin{abstract}
The present study seeks to identify a panorama on the subject of public transparency, which has been highlighted in the media in recent years and still presents a concept widely discussed in the literature. Thus, the objective of this study is to verify the evolution of the topic through the publication of articles in international journals on transparency in the public sector from 2007 to 2017. The research was established through the search of publications in periodicals based on Scopus. The analysis process was carried out through a process of organization, classification and analysis of a set of information and bibliometric data. To do so, this research is delineated as descriptive, bibliometric and qualitativequantitative analysis. A total of 540 articles were found with a predominance of published works in the United States, followed by Spain and the United Kingdom in second and third places respectively. In addition, the results demonstrate that the world literature on the subject of public transparency is consolidated, unlike the national literature that still needs to have greater advances in research in this area.
\end{abstract}

Keywords: Transparency. Public sector. Scientific publications. Governance. Bibliometric. 


\section{INTRODUÇÃO}

A propagação de fraudes $\mathrm{e}$ atos de corrupção encontram um ambiente propício quando da baixa transparência das informações dos atos da administração pública, ou seja, quanto menor for a transparência dos atos da administração pública maior a oportunidade de propagação de atos ilícitos. Segundo Sacramento (2007), a transparência pode ser vista como um instrumento capaz de contribuir para a redução de atos de corrupção. Sendo assim, torna-se importante a preocupação da administração pública em oportunizar meios e ferramentais para uma maior participação cidadã e maior transparência das informações dos atos praticados pela mesma.

Nesse processo de melhoria da transparência pública pode-se destacar a gestão de projetos com um fator cada vez mais relevante para a administração pública, uma vez que a sua utilização em alguns locais já trouxe resultados positivos $\mathrm{e}$ propiciou o melhoramento na Administração Pública (FURTADO et al., 2011; MARINI; MARTINS, 2014). Para Neto e Vacovski (2016), a implementação da gestão de projetos em instituições públicas tem trazido melhorias que demonstram uma maior qualidade à gestão e por consequência mais efetividade na transformação do que é planejado em resultados.

Logo, fica evidente a necessidade da transparência também na gestão dos projetos públicos. Mecanismos de monitoramento diários e com elevado nível de transparência podem ser uma opção facilitadora do controle social, o qual, através de uma maior participação consiga uma diminuição no nível de paralisações e aditivos que os projetos públicos. Assim, quem sabe os mesmos deixem de ser regra e passem a ser exceções. No momento, isso é algo que não vem acontecendo de uma forma geral nas instituições públicas e em especial com os projetos públicos do Brasil. Fato esse evidenciado pelo levantamento feito pela Revista Exame (2016), através do anuário Exame de Infraestrutura 2015 2016, das 10 maiores obras de infraestrutura que estavam em execução no país, onde verificou-se um aumento médio de $70 \%$ no orçamento em relação à previsão inicial.

Um dos motivos para esses problemas está na falta de um detalhamento adequado desde a concepção dos projetos. Um indício disso é o número expressivo de empreendimentos que sofrem alterações no valor do investimento. Segundo os dados da revista um em cada cinco projetos públicos no país sofrem uma revisão no orçamento. Outro fator é o problema da dilatação dos prazos dos projetos, cerca de $16 \%$ das obras tem seu prazo de conclusão dilatados por empecilhos ambientais, legais, técnicos ou de outra natureza (SEGALA, 2015).

Assim, torna-se evidente a necessidade de busca por mecanismos com o objetivo de incentivar a uma maior transparência na administração pública bem como também na gestão de seus projetos para assim auxiliar o cidadão a exercer seu direito e promover um maior controle social. Diante desse contexto, o objetivo do trabalho foi verificar a evolução do tema através da publicação de artigos em periódicos internacionais sobre transparência no setor público no período de 2007 a 2017.

$\mathrm{O}$ artigo tem sua sequência organizada de maneira que nas próximas seções é apresentado a importância da transparência na administração pública, o controle social e a transparência na 
administração pública, as características dos projetos públicos, o método de pesquisa, a análise dos dados e discussões e as considerações finais.

\section{FUNDAMENTAÇÃO TEÓRICA}

\subsection{Transparência na administração pública}

O Brasil tem passado, nos últimos tempos, por uma crise de valores relativos à moral e à ética. Fato esse que demonstra suas evidências através da descrença generalizada nas instituições públicas (MARTINS; VÉSPOLI, 2013). Nesse contexto, pode-se verificar uma necessidade cada vez maior quanto a promoção da transparência como forma fundamental para propiciar à condição de acesso as informações sobre os atos da administração pública. Em sua pesquisa Pires et al. (2013) destaca a importância da prática da transparência ativa por parte dos municípios, ou seja, divulgação espontânea dos dados, ações e serviços municipais ao cidadão. Os autores frisam ainda a necessidade necessária dos gestores realizem investimentos em sistemas de informações eficientes e buscarem capacitação de suas equipes para possibilitar um atendimento adequado, ágil e que atenda as expectativas e solicitações da sociedade.

Essa atuação de maneira aberta torna possível o desenvolvimento de uma gestão mais transparente. Segundo Medeiros et al. (2014) para a construção de uma verdadeira democracia, torna-se necessários que haja um acesso claro e transparente das informações públicas. Os autores destacam que o acesso a informação e a transparência podem não garantir um correto funcionamento da máquina pública, mas sem esses mecanismos é improvável que tal atividade ocorra sequer de maneira razoável.

Para Nascimento (2013), o objetivo principal dessas iniciativas, que visam permitir o livre acesso às informações dos atos realizados pelas instituições públicas, é proporcionar ferramentas que facilitem a participação social do cidadão como forma de exercer a cidadania de modo mais efetivo. Na mesma linha Cruz (2012), complementa afirmando que a transparência deve buscar garantir a todos os cidadãos acesso as informações que explicitam as ações a serem realizadas pelos representantes das instituições públicas bem como também as que estão em andamento e as que foram executadas em períodos anteriores.

Essa necessidade não se diferencia na gestão de projetos, políticas públicas e atividades nas instituições públicas. Sendo assim, para viabilizar uma gestão de projetos transparente é necessário que os projetos possibilitem uma maior participação do cidadão na escolha, gestão e controle. Entretanto, o que se tem visto como mais presente é um baixo número de organizações que disponibilizam canais de acesso a informações eficientes quanto ao andamento da gestão dos seus projetos. Em seu estudo Santos e Kreutz (2015) destacam a baixa transparência nos projetos públicos do PAC.

A gestão pública ainda é gerida através da solicitação, ou seja, carece de transparência ativa. O desejável na gestão de projetos seria a divulgação de forma espontânea e independente de qualquer solicitação todas as etapas do processo de elaboração e execução dos seus diferentes projetos. Esse cenário de baixa publicização acaba por criar um ambiente propício para propagação de atos de fraudes e corrupção no 
setor público. Fato esse que pode ser verificado através dos resultados apresentados pela pesquisa da ONG Transparência Internacional (TI). Segundo o "Índice de Percepção de Corrupção 2016", que lista os países baseados numa escala de zero (altamente corrupto) a 100 (muito transparente) o Brasil está no $79^{\circ}$ lugar entre 168 países participantes da pesquisa (Transparency International, 2017). Logo, o resultado demonstra o elevado nível de percepção de corrupção que o país se encontra.

Dessa forma, os dados parecem demonstrar a necessidade e a importância da transparência nas instituições públicas, pois com a mesma transparência será possível que haja um estimulo a participação e o controle social e consequentemente fortalecimento do processo de participação democrático e cidadã e assim quem sabe reduzir os índices de corrupção nos projetos públicos. A transparência é considerada no presente estudo como uma condição necessária para que a comunidade possa exercer seu controle social e participar do processo político da gestão dos projetos públicos, bem como da escolha dos mesmos. É necessário que haja acessibilidade e transparência na gestão para que seja possível alcançar uma mudança significativa na participação e controle social (JACOBI, 2003)

2.2 O Controle Social e a Transparência na Administração Pública

$\mathrm{Na}$ atualidade pode-se perceber o controle social como um instrumento de acompanhamento e controle das ações do estado, por parte do cidadão, o mesmo tem se tornado cada vez mais importante uma vez que tem tido maior visibilidade e importância no país nos últimos anos. Evidência disso é o interesse cada vez maior que as instituições públicas têm em buscar ações inovadoras para implementar na gestão pública com a intenção de combater diversos tipos de irregularidades dentro desse setor. Para Braga (2011), o Controle Social tem por objetivo buscar assegurar ou ampliar os direitos sociais do cidadão.

No entanto, não é possível ao cidadão desempenhar seu direito democrático de participação social se as instituições públicas não forem transparentes. Para Miragem (2011), a existência de um maior nível de informações disponíveis para o cidadão, estimula e qualifica igualmente a participação da população nos processos de conhecimento e decisão, ou seja, para ele trata-se de uma democracia informacional, que através da tecnologia traz uma redução da distância da administração pública e o cidadão.

Desse modo, pode-se evidenciar que o princípio da publicidade é um importante e prescindível instrumento para possibilitar o adequado processo de controle social, que pode ser visto como essencial em uma sociedade democrática. A literatura sobre a transparência aconselha que as organizações devem ser transparentes para aumentar o grau de confiança (RAWLINS, 2008). Dito isso, pode-se perceber que o controle social também pode ser visto como um mecanismo de prevenção da corrupção e como consequência fortalecendo o processo democrático da cidadania.

\subsection{Características dos projetos públicos}

Há algum tempo as diferenças na gestão de projetos entre o setor público e privado tem sido objeto de estudo de diversos pesquisadores. 
Na visão de Wirick (2009), o setor público é um ambiente conflituoso que envolve diversos atores com interesses diferentes e que apresentam pouca preocupação com o risco. Para Valle et al. (2007) o que se destaca como o grande desafio do setor público é achar e implementar mecanismos que possibilitem viabilizar um diálogo eficaz entre os objetivos e as ferramentas de gestão de projetos utilizadas pelo setor para o atendimento dos seus propósitos. Complementado essa perspectiva, Carneiro (2010), afirma que a esfera pública tem buscado implementar práticas de gerenciamento de projetos com mais intensidade e com o objetivo que a mesma seja uma ferramenta facilitadora para o atendimento das metas e consequentemente o alcance de um maior êxito na implementação das políticas públicas.

A administração pública se diferencia do setor privado justamente pelas suas características próprias e específicas, e em função disso existe uma necessidade de adoção de práticas diferenciadas para a gestão de projetos nas organizações públicas. Neste contexto, Crawford e Helm (2009), destacam como importante satisfazer múltiplos e imprevisíveis stakeholders como uma das especificidades do setor público. Essa diferença faz com que haja uma necessidade constante de uma comunicação mais efetiva, de formas de consulta e resposta a diferentes necessidades como comunidade, usuários, clientes, consumidores, outras agências de governo, aspectos regulatórios, políticos, imprensa, setores privados e outros atores competindo pelos seus interesses, demandas e objetivos.

Na perspectiva de Mainardes et al. (2010), outra especificidade das organizações públicas é a quantidade de partes interessadas que normalmente é superior as empresas tradicionais. $\mathrm{Na}$ visão do autor esse fato se evidencia porque a administração pública envolve uma maior diversidade de atividades e também um elevado número de pessoas, grupos e organizações, os quais têm interesse direto no resultado das atividades realizadas.

Outro fator de diferenciação, destacado na literatura, é a transparência e accountability. Enquanto que no setor público tem-se acentuado cada dia mais a necessidade de transparência e prestação de contas, na área privada não existe essa necessidade, pois as empresas existem para atender aos interesses de um indivíduo ou um grupo. Nessa mesma perspectiva, Costa Junior e Costa (2014), destacam a importância das instituições públicas para a questão da promoção da transparência e o estímulo ao controle social.

Neste sentido, diferente da área privada que não tem essa necessidade, pode-se verificar a existência de uma maior preocupação dos gestores públicos a disponibilização de canais, meios e portais de transparência para dessa forma poder possibilitar uma maior aproximação entre o cidadão e o Estado. Entretanto, segundo evidência de Khagram, De Renzio e Fung (2013), as relações entre transparência fiscal, participação e prestação de contas são, de maneira geral, fracas, erráticas e incompletas.

Em seus estudos Boyne (2002), realizou uma revisão de publicações de pesquisas que tiveram como foco identificar diferenças apresentadas pelas empresas privadas e órgãos públicos. $\mathrm{O}$ autor destaca a existência de diferenças entre as organizações públicas e privadas tem levado ao surgimento de barreiras no que se refere a questão da transferência de técnicas de gestão do setor privado para o público 
Assim, Boyne (2002) destaca em seu trabalho o fato da existência de diferenças significativas. As mesmas são tanto em função dos ambientes organizacionais, dos objetivos, de suas estruturas e seus valores de gestão. Essas diferenças fazem com que haja dificuldade na exportação das técnicas de gestão com sucesso de um setor para outro.

Na mesma perspectiva, Zwicker, Fettke e Loos (2010) trazem sua complementação afirmando que as ações administrativas são afetadas diretamente pelos princípios burocráticos. Para o autor essa passa a ser uma das principais diferenças entre a administração pública e a privada.

É perceptível a existência de interesse das organizações públicas e dos avanços já conquistado, entretanto, os gestores ainda não conseguem minimizar os riscos no processo de gestão de projetos nas organizações públicas em geral. Isso pode ser evidenciado facilmente através da interminável lista de obras paradas e projetos não concluídos no setor público (SEGALA, 2015).

\section{MÉTODO DE PESQUISA}

A abordagem metodológica adotada nesta revisão sistemática da literatura mesclas técnicas de bibliometria, análise de conteúdo e nuvem de palavras. As técnicas de bibliometria permitiram estabelecer tendências em termos do número de publicações e citações ao longo do tempo, principais autores e obras. Uma pesquisa bibliométrica tem por objetivo medir e avaliar a produção científica já pesquisada. Logo, essa técnica traz a possibilidade de realizar um mapeamento da produtividade científica de periódicos e representação da informação. Assim, pode-se realizar-se também uma observação da evolução da literatura sobre o assunto no decorrer dos anos (ARAUJO et al., 2000). Realizou-se a escolha do método bibliométrico em função do interesse verificar as atividades de produção no campo da transparência pública.

Ademais, uma análise qualitativa sobre alguns dos principais e mais citados trabalhos encontrados foi realizado, com o objetivo de verificar os principais temas abordados na pesquisa aplicou-se o método de nuvem de palavras, que agrupa as palavras e as organiza graficamente em função da sua frequência. Quanto as etapas de coleta de dados, pode-se destacar que o procedimento foi realizado por meio de uma pesquisa de artigos científicos publicados em periódicos.

Esse processo de busca-se foi realizado de novembro de 2017, na base de dados do Scopus, foram utilizadas as palavras-chave de busca "Transparency" AND "Public" AND "Sector", nesta ordem, entre aspas, para pesquisar o termo por inteiro, e não as palavras em separado, que deveriam constar no título, resumo ou palavraschave dos artigos. Além disso, utilizou-se o filtro para tipo de documentos "Article" e estabeleceuse uma restrição temporal de 2007 a 2017, sendo que a consulta foi feita nas subáreas: "Business, Management e Accounting" e "Social Sciences".

Com a aplicação dos filtros relacionados aos parâmetros da pesquisa, foram encontrados 540 artigos sobre o tema. Ato contínuo, os dados relativos ao tema foram tabulados no software Excel, de forma a evidenciar as características das publicações acadêmicas mais citadas. Para a análise das publicações por ano, por periódico, por instituição, e por país, foram utilizadas as 
ferramentas de tabulação ofertadas pela própria base de dados do Scopus, onde foi possível a elaboração de gráficos com tais informações.

$\mathrm{Na}$ segunda etapa, visando aprofundar o tema pesquisado, selecionou-se três artigos mais citados para fazer uma análise qualitativa sobre quais os assuntos abordados nos artigos de maior impacto cientifico. A seleção dos três artigos mais citados internacionalmente justifica-se, pois se entende que os mais citados são os que mais geram contribuições para os estudos da área, e por isso fez-se uma análise destes. Por fim, como o objetivo de verificar os temas mais abordados pelos artigos utilizou-se o método de nuvem de palavras.

\section{DISCUSSÃO E ANÁLISE DOS RESULTADOS}

\author{
4.1 Análise das de publicações em \\ journals
}

Ao todo, 160 journals foram identificados com publicações relacionadas à transparência no setor público. Os resultados demonstram que de 2007 para 2008 houve um acentuado declínio. No entanto em 2009 até 2012 percebe-se uma tendência de elevação no número de artigos publicados. Já em 2013 verifica-se novamente uma queda bastante elevada. Entretanto, em 2014 ocorre uma elevação novamente e nos anos seguintes, 2015, 2016 e 2017 percebe-se uma tendência constante com pequena variação no número de publicações.

Os resultados indicam que foram publicados 30 artigos no ano de 2007; 15 artigos no ano de 2008; 36 artigos no ano de 2009; 40 artigos no ano de 2010; 51 artigos no ano de 2011; 60 artigos no ano de 2012; 36 artigos no ano de 2013; 69 artigos no ano de 2014; 67 artigos no ano de 2015, 68 artigos no ano de 2016 e 2017. Observa-se que as publicações relacionadas a temática transparência no setor público estão em uma crescente, o que traz evidências de um crescente interesse pelos pesquisadores em desenvolverem pesquisas no tema e na área relacionada.

No período dos últimos seis anos a amostra de 540 artigos foi citada 4123 vezes, ou seja, uma média de 7,63 citações por publicação. Já os 10 artigos mais citados possuem 1147 citações ao todo, perfazendo uma média de 114,7 citações por artigo.

Em relação aos periódicos, conclui-se que o periódico internacional que mais publicou sobre transparência no setor público é o Internacional Review Of Administrative Sciences, com 14 artigos publicados e o Government Information Quarterly e International Jounal of Public Administration, com 10 publicações ficaram empatados em segundo lugar.

Referente as instituições, pode ser observado que a Universidade de Granada da Espanha se destacou com a principal universidade em publicações sobre o tema. Em segundo lugar ficou a Universidade de Almeria, também da Espanha, e a Universidade de Toronto, do Canadá, ficou em terceiro lugar, ou seja, essas podem ser consideradas como as principais universidades em nível de publicações de artigos científicos sobre a transparência no setor público.

O país que mais publicou artigos sobre o tema com 86 artigos foi o Estados Unidos, seguido pela Espanha com 77 artigos, e, na sequência, pelo Reino Unido com 68 artigos. 
Logo, parece que os resultados indicam certa supremacia desses três países em relação aos demais no quesito publicações sobre o tema

Destaca-se que são apresentadas no gráfico apenas os dez países principais em número de publicações. O Brasil aparece na lista em sétimo lugar com dezenove publicações, ou seja, ainda existe muito a ser estudado sobre o tema no país.

4.2 Caracterização de algumas publicações mais citadas

Entende-se que o número de citações de um artigo é um fator que influencia o desenvolvimento da área em questão, considerando que os pesquisadores estão se embasando numa mesma fonte de informação para contribuir para os estudos da área. Desta forma, será analisado a seguir o tema específico dos três mais citados.

O trabalho de Bonsón, E., Torres, L., Royo, S., Flores, F. (2012), intitulado Local egovernment 2.0: Social media and corporate transparency in municipalities, com 276 citações, destaca a contribuição potencial da internet para melhorar a interatividade, transparência e abertura de entidades do setor público e para promover novas formas de prestação de contas. $\mathrm{O}$ trabalho tem por objetivo fornecer um visão geral sobre o uso da Web 2.0 e ferramentas de mídia social nos governos locais da União Europeia a fim de determinar se os governos estão usando essas tecnologias para aumentar a transparência e a participação eletrônica. Como resultados a pesquisa os autores destacam que a maioria dos governos está utilizando das ferramentas pesquisadas para aumentar a transparência, apesar de haver um alto nível de heterogeneidade no uso das mesmas.

No segundo artigo de Picazo-Vela, S., Gutiérrez-Martínez, I., Luna-Reyes, L.F. (2012), intitulado Understanding risks, benefits, and strategic alternatives of social media applications in the public sector, com 119 citações os autores destacam que as organizações governamentais estão cada vez mais experimentando utilizar as as redes sociais para comunicar com os seus constituintes. No entanto, existema alguns especialistas que vêem nestes meios um poderoso conjunto de ferramentas para reinventar relações governo-cidadão. Como resultados é destacado pelos autores alguns prontos: a) que a participação dos governos nas mídias sociais pode resultar na melhoria da comunicação e participação cidadã, mais transparência e transferência de melhores práticas entre agências governamentais; b) que é necessária uma boa estratégia de implementação para realizar esses benefícios e para evitar riscos; e c) que a implementação das redes sociais destaca a importância da atualização leis e regulamentos, e faz-se necessário uma promoção de mudanças na cultura do governo e práticas organizacionais.

Já o trabalho de Meijer, A. (2009), intitulado Understanding modern transparency, com 99 citações, apresenta uma abordagem sobre os defensores e opositores quanto ao debate sobre a transparência ser mediada por computador e como ela traz um efeito positivo na confiança no setor público. A pesquisa teve por objetivo buscar uma melhor na compreensão da transparência, apresentando três perspectivas: um pré-moderno, perspectiva moderna e pós-moderna, e analisando os pressupostos básicos de essas perspectivas sobre transparência. Com base nessa análise, o 
autor defende a diversidade em sistemas de transparência para maximizar os efeitos sobre a confiança social.

\subsection{Método de nuvem de palavra}

A terceira etapa de análise desta pesquisa foi desenvolvida através da utilização do método de nuvem de palavras. Utilizou-se essa técnica com o objetivo de verificar quais os temas mais abordados nas pesquisa realizadas e que foram mais citadas. Para tanto utilizou-se as palavras chaves dos 10 artigos mais citados.

Figura 1 - Nuvem de palavras artigos mais citados

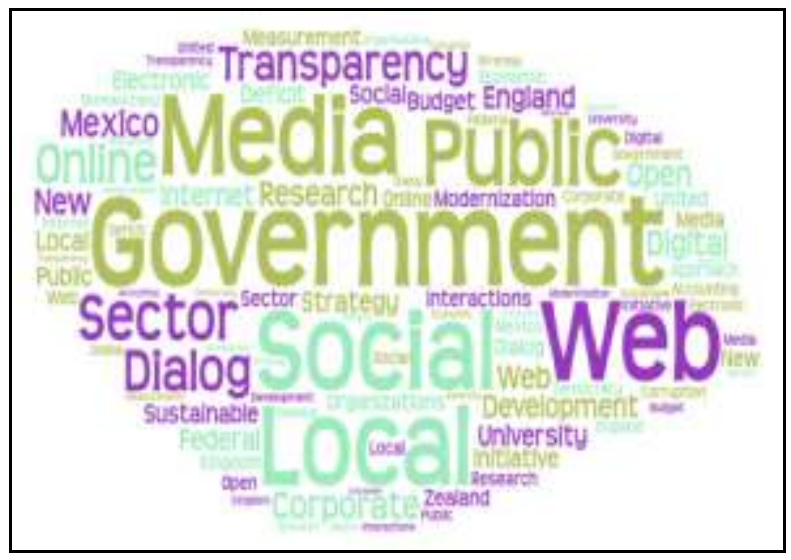

Fonte: Desenvolvido pelos autores.

A figura 1 mostra a nuvem gerada para os temas relacionados a transparência no setor público referente a amostra dos 10 artigos mais citados na presente pesquisa. Nota-se na figura que as palavras são posicionadas aleatoriamente de tal forma que as palavras mais frequentes aparecem maiores que as outras, demonstrando, assim, seu destaque na análise da pesquisa.

Pode-se evidenciar que as palavras que obtiveram maior destaque formam government, mídia, social, local, web e public. Logo, os resultados parecem indicar que as pesquisas de maior representatividade no meio acadêmico, e que se referem ao tema aqui estudado, tem direcionado maior parte do seu foco para a utilização de mídias sociais nas instituições públicas como uma ferramenta para fortalecimento a transparência e por consequência um melhor relacionamento com o cidadão.

\section{CONSIDERAÇÕES FINAIS}

O presente estudo objetivou identificar um panorama das publicações de artigos científicos em periódicos internacionais que tratam do tema transparência aplicada ao setor público nos últimos onze anos. O período em análise foi entre os anos de 2007 e 2017, em artigos disponíveis na base de dados Scopus.

Os resultados deixam evidente que existe uma tendência em ascendência, pois o número de publicações relacionadas ao tema em estudo tem apresentado uma elevação nos últimos onze anos. $\mathrm{Na}$ Espanha ficam duas das instituições que mais publicam sobre o tema, entretanto, o país que mais publica artigos sobre o tema transparência no setor público são os Estados Unidos.

Cabe destacar ainda que apesar do Brasil não ter apresentado uma grande relevância em número de publicações internacionais sobre o tema, está em sétimo lugar dentre os 10 países que mais publicam, logo fica evidente que esse tema ainda é algo a ser bastante explorado em pesquisas futuras no país.

Por fim, tem-se que a questão da transparência aplicada ao setor público é um tema quem vem crescendo em número de publicações a nível mundial. Destaca-se como limitação dessa pesquisa o fato da abordagem se dar apenas por artigos e utilização de uma base única do Scopus. 
Além disso, sugere-se para estudos futuros a possibilidade de ampliação para outros documentos bem como também utilização de outras bases de busca de periódicos.

\section{REFERÊNCIAS}

ARAUJO, W. T. et al. Meta-análise das dissertações do curso de mestrado ciência da informação UFPB: 1990-1999. Revista Informação e Sociedade: estudos, João Pessoa, v. 10, n. 1, p. 1-11, 2000.

BRAGA, M. V. de A. A auditoria governamental como instrumento de promoção da transparência. Revista Jus Navigandi, Teresina, ano 16, n. 2900, 2011.

BOYNE, G. A. Public and private management: what's the difference? Journal of Management Studies, v. 39, n.1, p. 97/122, 2002.

CARNEIRO, M. F. S. Gestão Pública: o papel do planejamento estratégico, gerenciamento de portfólio, programas e projetos e dos escritórios de projetos na modernização da gestão pública. Rio de Janeiro, RJ: Brasport, 2010.

COSTA JÚNIOR, A. G.; COSTA C. E. M. A Comunicação como Efetivação da Transparência na Gestão Pública: um estudo de caso em uma Prefeitura Municipal no interior do Nordeste. Revista Tematica, a. x, n. 2, p. 1-40, 2014.

CRAWFORD, L. H; HELM, J. Government and governance: The value of project management in the public sector. Project Management Journal, v. 40, n. 1, p. 73-87, 2009.

CRUZ, C.F. Transparência da gestão pública municipal: um estudo a partir dos portais eletrônicos dos maiores municípios brasileiros.

Rev. Adm. Pública, v. 46, n.1, p.153-176, 2012.

FURTADO, M. A. P. FORTUNATO, G.; TEIXEIRA. A percepção dos gestores da área pública sob a política de gerenciamento de projetos. Revista Eletrônica Sistemas \& Gestão v. 6, n. 2, p. 167-183, 2011.

JACOBI, P. Educação ambiental, cidadania e sustentabilidade. Caderno de Pesquisa, n.118, p. 189-206, 2003.
KHAGRAM, S.; DE RENZIO, P.; FUNG, A. Overview and synthesis: the political economy of fiscal transparency, participation, and accountability around the world. Open budgets: The political economy of transparency, participation, and accountability, 1-50, 2013.

MAINARDES, E. W.; ALVES, H.; RAPOSO, M.; DOMINGUES, M. J.. Categorização por importância dos stakeholders das universidades. Revista Ibero-Americana de Estratégia, São Paulo, v. 9, n. 3, p. 5-40, 2010.

MARINI, C.; MARTINS, H. F Todos por Pernambuco em tempos de governança: conquistas e desafios. Recife: Instituto Publix, 2014.

MARTINS, P. L.; VÉSPOLI, B. S. O Portal da Transparência como Ferramenta para a Cidadania e o Desenvolvimento. Revista de Administração da Fatea, v. 6, n. 6, p. 93-102, 2013.

MEDEIROS, S. A.; MAGALHÃES, R.; PEREIRA, J. R. Lei de acesso à informação: em busca da transparência e do combate à corrupção. Revista Informação, v. 19, n. 1, p. 55 - 75, 2014.

MIRAGEM, B. A nova administração pública e o direito administrativo. São Paulo, SP: Revista dos Tribunais, 2011.

NASCIMENTO, L.P. A Transparência dos Portais Brasileiros de Transparência Pública: Um Estudo de Três Casos. RAF Revista de Administração da Fatea, v. 6, n. 6, p. 93-102, 2013.

NETO, A. N. A.; VACOVSKI, E. O gerenciamento de projetos e sua importância para a qualidade e a efetividade no setor público.

Revista Caderno Gestão Pública. v. 8, n. 5. 2016.

PIRES, A. M.; SCHERER, F. L.; SANTOS, M. B.; CARPES, A. M. Transparência da gestão pública municipal: um estudo dos municípios de Santa Maria e Novo Hamburgo / RS. Revista Estudos do CEPE, Santa Cruz do Sul, n. 38, p. 131-160, 2013.

RAWLINS, B.L. Measuring the relationship between organizational transparency and employee trust. Public Relations Journal, v. 2, n. 2, p. 1-21, 2008.

SACRAMENTO, A. R. S.; PINHO, J. A. G. Transparência na administração pública: o que 
mudou depois da lei de responsabilidade fiscal? Um estudo exploratório em seis municípios da região metropolitana de Salvador. Revista de Contabilidade da UFBA, v.1, n.1, p. 48-61, 2007.

SANTOS, C. H. S; KREUTZ, R. R. O ciclo de vida e a transparência dos projetos do PAC no Setor Público do Brasil. Revista Instituciones y Competitividad, v. 2, n. 2, p. 31-45, 2015.

SEGALA, M. Anuário Exame de Infraestrutura 2015-2016. Revista Exame, ed. 1099, a. 49, n. 19, p. 106-117, 2015.

TI (Transparency International). Índice de Percepção de Corrupção 2016. Berlin Germany (2017). Disponível em <http://transparency.org/cpi>. Acessado em novembro 2017

\section{WIRICK, DW. Puclic-Sector Project}

Management: Meeting the Challenges and Achieving the Results, John Wiles \& Sons, 2009.

VALLE, A. B.; Soares, C. A. P.; Finocchio JR., J.; Silva, L. S. F. Fundamentos do gerenciamento de projetos. Rio de Janeiro, RJ: FGV, 2007.

ZWICKER, J.; FETTKE, P.; LLOOS, P. Business Process Maturity in Public Administrations. In: Handbook on Business Process Management 2: Strategic Alignment, Governance, People and Culture (International Handbooks on Information Systems). BROCKE, Jan Vom (Editor);

ROSEMANN, Michael (Editor). Berlin: Springer, p. $369-400,2010$.

\begin{abstract}
Rafael Rudolfo Kreutz
Mestrando em Gestão de Organizações Públicas da Universidade Federal de Santa Maria (UFSM). Graduação em administração de empresas pela UFSM, MBA em Administração e Finanças pela Uninter e Especialização em Gestão Pública pela Universidade Estadual do Rio Grande do Sul (UERGS). Atualmente Analista Administrador pela Universidade Estadual do Rio Grande do Sul (UERGS), e Diretor executivo da Revista Eletrônica Científica da UERGS.
\end{abstract}

Nelson Guilherme Machado Pinto
Professor Adjunto da Universidade Federal de Santa
Maria (UFSM) lotado no Departamento de
Administração no Campus Palmeira das Missões e
Professor do Programa de Pós-Graduação em Gestão
de Organizações Públicas da Universidade Federal de
Santa Maria (PPGOP/UFSM). Doutor em
Administração pela UFSM na linha de pesquisa de
Economia, Controle e Finanças. Mestre em
Administração pela UFSM na linha de pesquisa de
Economia, Controle e Finanças. Bacharel em
Administração pela UFSM.

